\title{
Chemical Constituents from Mentha haplocalyx Briq. (Mentha canadensis L.) and Their a-Glucosidase Inhibitory Activities
}

\author{
Xiao-Feng He ${ }^{1,2,3} \cdot$ Chang-An Geng ${ }^{1,2} \cdot$ Xiao-Yan Huang ${ }^{1,2} \cdot$ Yun-Bao Ma ${ }^{1,2} \cdot$ Xue-Mei Zhang ${ }^{1,2} \cdot J^{-}$-Jun Chen ${ }^{1,2,3}(0)$
}

Received: 14 March 2019 / Accepted: 17 April 2019 / Published online: 29 April 2019

(c) The Author(s) 2019

\begin{abstract}
Mentha haplocalyx (Mentha canadensis) is widely used as a medicinal plant in traditional Chinese medicine, and the extracts of its aerial parts are found to significantly inhibit the activity of $\alpha$-glucosidase with an $\mathrm{IC}_{50}$ value of $21.0 \mu \mathrm{g} / \mathrm{mL}$. Bioactivity-guided isolation of the extracts afforded two new compounds (1 and 2), together with 23 known ones (3-25). Their structures were established by extensive spectroscopic analyses (1D and 2D NMR, MS, IR and UV). Compounds 1-17 and 21-25 were evaluated for their $\alpha$-glucosidase inhibitory activities. Compound $\mathbf{1 1}$ was the most active ones with an $\mathrm{IC}_{50}$ values of $83.4 \mu \mathrm{M}$. These results verify the $\alpha$-glucosidase inhibitory activity of M. haplocalyx (M. canadensis) and specify its active compounds for the first time.
\end{abstract}

\section{Graphical Abstract}
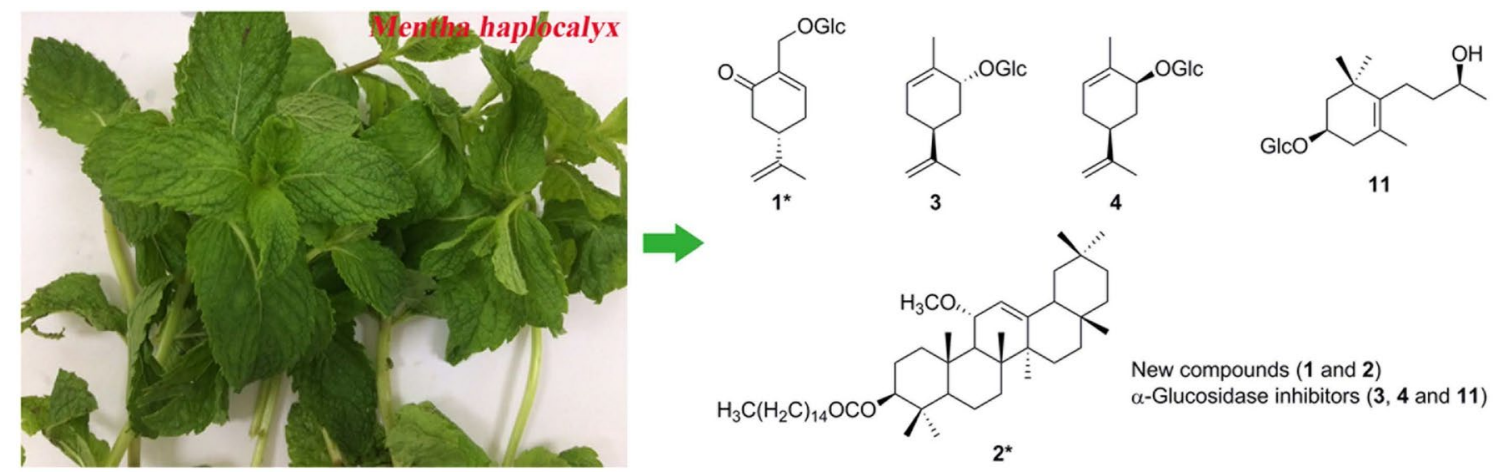

New compounds ( 1 and 2 ) $\alpha$-Glucosidase inhibitors $(3,4$ and 11$)$

Keywords Mentha haplocalyx Briq. (Mentha canadensis L.) $\cdot$ Lamiaceae $\cdot \alpha$-Glucosidase inhibitor

Electronic supplementary material The online version of this article (https://doi.org/10.1007/s13659-019-0207-0) contains supplementary material, which is available to authorized users.

Ji-Jun Chen

chenjj@mail.kib.ac.cn

1 State Key Laboratory of Phytochemistry and Plant Resources in West China, Kunming Institute of Botany, Chinese Academy of Sciences, Kunming 650201, People's Republic of China

2 Yunnan Key Laboratory of Natural Medicinal Chemistry, Kunming 650201, China

3 University of Chinese Academy of Sciences, Beijing 100049, China

\section{Introduction}

Diabetes mellitus is a chronic disease caused by inherited or acquired deficiency in insulin secretion and by decreased responsiveness of the organs to secreted insulin [1]. Such a deficiency results in an increased blood glucose level and in turn damages body systems including blood vessels and nerves [2]. It is one of the most serious diseases worldwide and developing with an increase in obesity and ageing [3]. An efficiently therapeutic approach is to retard absorption of glucose through the inhibition of carbohydrate-hydrolysing enzymes such as $\alpha$-amylase and $\alpha$-glucosidase in the digestive organs [4]. Clinically, acarbose and voglibose have been 
used as effective $\alpha$-glucosidase inhibitors to delay glucose absorption [5].

Mentha haplocalyx Briq. (Mentha canadensis L.), a perennial herbaceous plant of the family Lamiaceae, is widely distributed in southwest of China and popularly used in food, cosmetics and medicines. As a traditional Chinese medicine, it is clinically used to treat diseases in the nerve center, breath, procreation and digestive systems [6]. Pharmacological studies of $M$. haplocalyx (M. canadensis) revealed various biological activities, such as antimicrobial, antiinflammatory, antioxidant, antitumor, gastrointestinal protective, and hepatoprotective activities [7]. A large number of volatile compounds were reported from $M$. haplocalyx (M. canadensis), as well as a few polyphenolic acids, flavonoids, monoterpenoids, and glycosides, which might contribute to the medicinal benefits of this plant [8].

Several findings have depicted the potential antidiabetic capability of genus Mentha. M. piperita could alleviate hyperglycemia induced by streptozotocin-nicotinamideinduced type 2 diabetes in rats, and cause a reduction of glycemia in human $[9,10]$. However, no report has referred to the active compounds of Mentha responsible for its antidiabetic capability. Our preliminary bioassay revealed that extracts of the aerial parts of M. haplocalyx (M. canadensis) exhibited significant $\alpha$-glucosidase inhibitory activity with an $\mathrm{IC}_{50}$ value of $21.0 \mu \mathrm{g} / \mathrm{mL}$. Subsequently, two new compounds (1 and 2) (Fig. 1) and 23 known ones (3-25) were isolated and identified through bioactivity-guided fractionation. This paper described the isolation, identification, and $\alpha$-glucosidase inhibitory activity evaluation of these compounds.

\section{Results and Discussion}

\subsection{Structural Identification}

Compound 1 was obtained as colorless caramelized solid, with the molecular formula of $\mathrm{C}_{16} \mathrm{H}_{24} \mathrm{O}_{7}$ from its HRESIMS at $m / z 351.1404[\mathrm{M}+\mathrm{Na}]^{+}$. The IR absorptions suggested the existence of a hydroxyl group $\left(3427 \mathrm{~cm}^{-1}\right)$

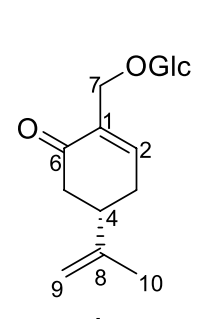

1

Fig. 1 The structures of new compounds 1 and 2 from M. haplocalyx and an $\alpha, \beta$-unsaturated carbonyl group $\left(1635 \mathrm{~cm}^{-1}\right)$. The ${ }^{13} \mathrm{C}$ NMR (DEPT) data (Table 1) displayed sixteen carbon signals, including one methyl, five methylenes, seven methines, and three quaternary carbons. Among them, a glucose unit $\left(\delta_{\mathrm{C}} 104.1,75.2,78.2,71.7,78.2,62.8\right)$ and an $\alpha, \beta$-unsaturated carbonyl group $\left(\delta_{\mathrm{C}} 136.6,149.4\right.$, 201.0) were observed. ${ }^{1} \mathrm{H}$ NMR data (Table 1) exhibited a singlet methyl proton $\left(\delta_{\mathrm{H}} 1.78, \mathrm{H}-10\right)$, a trisubstituted olefinic proton $\left(\delta_{\mathrm{H}} 7.23, \mathrm{H}-2\right)$, and one terminal double bond proton $\left(\delta_{\mathrm{H}} 4.82, \mathrm{H}-9 \mathrm{a} ; 4.80, \mathrm{H}-9 \mathrm{~b}\right)$. The glucose unit protons $\left[\delta_{\mathrm{H}} 4.30\left(\mathrm{~d}, J=7.8 \mathrm{~Hz}, \mathrm{H}-1^{\prime}\right) ; 3.19-3.85(6 \mathrm{H}\right.$, $\left.\mathrm{H}-2^{\prime}-\mathrm{H}-6^{\prime}\right)$ ] were also present. All the above information suggested that compound $\mathbf{1}$ was a menthane-monoterpene glycoside. Apart from the glucose unit, the NMR data of 1 closely resembled those of the reported compound $(R)$ 7-hydroxycarvone [11]. Whereas the major differences between their NMR signals at C-7 position $\left[\delta_{\mathrm{H}} 4.49,4.28\right.$ and $\delta_{\mathrm{C}} 67.0$ for $1 ; \delta_{\mathrm{H}} 4.27$ and $\delta_{\mathrm{C}} 61.8$ for $(R)$-7-hydroxycarvone] suggested that 7-OH in 1 might be glycosylated, which was further confirmed by the HMBC correlation of H-7 ( $\delta_{\mathrm{H}} 4.99$ and 4.28) with C-1' $\left(\delta_{\mathrm{C}} 104.1\right)$ (Fig. 2). The specific rotation of $\mathbf{1}\left([\alpha]_{\mathrm{D}}^{20}-22.0\right)$ was opposite to (4R)-7-hydroxyisopiperitenone7- $O$ - $\beta$-D-glucopyranoside $\left([\alpha]_{\mathrm{D}}^{20}+53.9^{\circ}\right)$, and similar to the $(4 S)$-7-hydroxyisopiperitenone 7- $O$ - $\beta$-D-glucopyranoside $\left([\alpha]_{\mathrm{D}}^{20}-14.4\right)[12]$, tentatively determining its $(4 S)$-configuration. Therefore, compound 1 was elucidated as (4S)-7-hydroxy-carvone 7-O- $\beta$-D-glucopyranoside.

Compound 2, white powder, had a molecular formula of $\mathrm{C}_{47} \mathrm{H}_{82} \mathrm{O}_{3}$ from its HREIMS $\left(\mathrm{m} / z 694.6258[\mathrm{M}]^{+}\right)$. The ${ }^{13} \mathrm{C}$ NMR (DEPT) data (Table 2) displayed 47 carbon signals, including ten methyls, 23 methylenes, six methines and eight quaternary carbons. Among them, a methoxyl $\left(\delta_{\mathrm{C}} 53.4\right)$, two oxy-methines $\left(\delta_{\mathrm{C}} 80.4, \mathrm{C}-3 ; 75.8, \mathrm{C}-11\right)$, a trisubstituted double bond $\left(\delta_{\mathrm{C}} 121.8,149.7\right)$ were showed. A palmitoyl group $\left(\delta_{\mathrm{C}} 173.7, \mathrm{C}-1^{\prime} ; 34.7, \mathrm{C}-2^{\prime} ; 25.2, \mathrm{C}-3^{\prime}\right.$; 29.2-29.7, C-4'-C-13'; 31.9, C-14'; 22.7, C-15'; 14.1, C-16') was also present. The ${ }^{1} \mathrm{H}$ NMR data (Table 2) exhibited eight singlet methyls $\left(\delta_{\mathrm{H}} 0.85-1.21\right)$, a triplet methyl $\left(\delta_{\mathrm{H}}\right.$ $0.88, \mathrm{t}, J=7.8 \mathrm{~Hz})$, a methoxyl $\left(\delta_{\mathrm{H}} 3.25\right)$, and a trisubstituted olefinic proton $\left(\delta_{\mathrm{H}} 5.32, \mathrm{~d}, J=3.0 \mathrm{~Hz}, \mathrm{H}-12\right)$. These NMR data suggested that $\mathbf{2}$ was an oleanane-type triterpene fused with a palmitoyl group, and closely resembled those of the reported compound $(3 \beta, 11 \alpha)$-11-hydroxy-olean-12en-3-yl palmitate [13]. The major difference was that the NMR signals at $\mathrm{C}-11$ of the known compound $\left(\delta_{\mathrm{H}} 4.50\right.$; $\left.\delta_{\mathrm{C}} 81.6\right)$ were upfield shifted in $2\left(\delta_{\mathrm{H}} 3.89 ; \delta_{\mathrm{C}} 75.8\right)$ and a methoxyl group $\left(\delta_{\mathrm{H}} 3.25 ; \delta_{\mathrm{C}} 53.4\right)$ further appeared, indicating the hydroxyl group at $\mathrm{C}-11$ was methylated in $\mathbf{2}$, which was further confirmed by the HMBC (Fig. 2) correlation of OMe $\left(\delta_{\mathrm{H}} 3.25\right)$ with $\mathrm{C}-11\left(\delta_{\mathrm{C}} 75.8\right)$. Finally, compound 2 was established as $(3 \beta, 11 \alpha)$-3-hydroxy-11 $\alpha$-methoxy-olean12-en-3-yl palmitate. 
Table $1{ }^{1} \mathrm{H}$ NMR $(600 \mathrm{MHz}$, $\left.\mathrm{CD}_{3} \mathrm{OD}\right)$ and ${ }^{13} \mathrm{C}$ NMR $\left(150 \mathrm{MHz}, \mathrm{CD}_{3} \mathrm{OD}\right)$ data for compound 1

\begin{tabular}{llrll}
\hline No & $\delta_{\mathrm{H}}$ & $\delta_{\mathrm{C}}$ & ${ }^{1} \mathrm{H}-{ }^{1} \mathrm{H}$ COSY & $\mathrm{HMBC}$ \\
\hline $\mathbf{1}$ & & $136.6 \mathrm{~s}$ & & \\
$\mathbf{2}$ & $7.23 \mathrm{~m}$ & $149.4 \mathrm{~d}$ & $\mathrm{H}-3 \mathrm{a}, 3 \mathrm{~b}, 7 \mathrm{a}, 7 \mathrm{~b}$ & $\mathrm{C}-1,3,4,6,7$ \\
$\mathbf{3 a}$ & $2.67 \mathrm{~m}$ & $32.2 \mathrm{t}$ & $\mathrm{H}-2,3 \mathrm{~b}$ & $\mathrm{C}-1,2,4,5$ \\
$\mathbf{3 b}$ & $2.41 \mathrm{ddq}(18.0,10.2,1.8)$ & & $\mathrm{H}-2,3 \mathrm{a}$ & $\mathrm{C}-1,2,4,5$ \\
$\mathbf{4}$ & $2.74 \mathrm{ddd}(16.2,10.2,4.8)$ & $43.6 \mathrm{~d}$ & $\mathrm{H}-5 \mathrm{a}, 5 \mathrm{~b}$ & $\mathrm{C}-3,5,6,8,9,10$ \\
$\mathbf{5 a}$ & $2.50 \mathrm{ddd}(16.2,5.4,1.2)$ & $44.2 \mathrm{t}$ & $\mathrm{H}-4$ & $\mathrm{C}-3,4,6,8$ \\
$\mathbf{5 b}$ & $2.48 \mathrm{~m}$ & & $\mathrm{H}-4$ & $\mathrm{C}-3,4,6,8$ \\
$\mathbf{6}$ & & $201.0 \mathrm{~s}$ & & $\mathrm{C}-1,2,6,1^{\prime}$ \\
$\mathbf{7 a}$ & $4.49 \mathrm{dq}(13.2,1.2)$ & $67.0 \mathrm{t}$ & $\mathrm{H}-7 \mathrm{~b}$ & $\mathrm{C}-1,2,6,1^{\prime}$ \\
$\mathbf{7 b}$ & $4.28 \mathrm{dd}(13.2,1.2)$ & & $\mathrm{H}-7 \mathrm{a}$ & $\mathrm{C}-4,8,10$ \\
$\mathbf{8}$ & & $148.3 \mathrm{~s}$ & & $\mathrm{C}-4,8,10$ \\
$\mathbf{9 a}$ & $4.82 \mathrm{~m}$ & $111.3 \mathrm{~s}$ & $\mathrm{H}-10$ & $\mathrm{C}-4,8,9$ \\
$\mathbf{9 b}$ & $4.80 \mathrm{~m}$ & & & $\mathrm{C}-7,2^{\prime}, 3^{\prime}, 5^{\prime}$ \\
$\mathbf{1 0}$ & $1.78 \mathrm{~s}$ & $20.7 \mathrm{q}$ & $\mathrm{H}-9 \mathrm{a}$ & $\mathrm{C}-3^{\prime}$ \\
$\mathbf{1}^{\prime}$ & $4.30 \mathrm{~d}(7.8)$ & $104.1 \mathrm{~d}$ & $\mathrm{H}-2^{\prime}$ & $\mathrm{C}-2^{\prime}, 4^{\prime}$ \\
$\mathbf{2}^{\prime}$ & $3.19 \mathrm{dd}(9.6,8.4)$ & $75.2 \mathrm{~d}$ & $\mathrm{H}-1^{\prime}, 3^{\prime}$ & $\mathrm{C}-5^{\prime}, 6^{\prime}$ \\
$\mathbf{3}^{\prime}$ & $3.34 \mathrm{t}(9.0)$ & $78.2 \mathrm{~d}$ & $\mathrm{H}-2^{\prime}$ & $\mathrm{C}-1^{\prime}, 4^{\prime}$ \\
$\mathbf{4}^{\prime}$ & $3.24 \mathrm{~m}$ & $71.7 \mathrm{~d}$ & $\mathrm{H}-5^{\prime}$ & $\mathrm{C}-4^{\prime}, 5^{\prime}$ \\
$\mathbf{5}^{\prime}$ & $3.28 \mathrm{t}(9.6)$ & $78.2 \mathrm{~d}$ & $\mathrm{H}-4^{\prime}, 6^{\prime} \mathrm{a}, 6^{\prime} \mathrm{b}$ & $\mathrm{C}-4^{\prime}, 5^{\prime}$ \\
$\mathbf{6}$ & $3.85 \mathrm{dd}(12.0,1.8)$ & $62.8 \mathrm{t}$ & $\mathrm{H}-5^{\prime}, 6^{\prime} \mathrm{b}$ & \\
$\mathbf{6} \mathbf{\prime} \mathbf{b}$ & $3.66 \mathrm{dd}(12.0,5.4)$ & & $\mathrm{H}-5^{\prime}, 6^{\prime} \mathrm{a}$ & \\
\hline
\end{tabular}

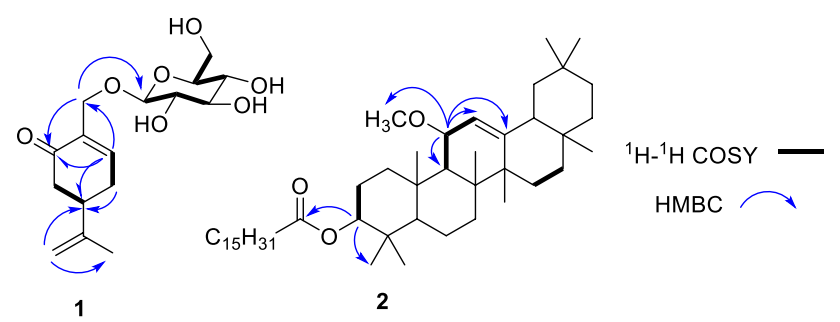

Fig. 2 Key ${ }^{1} \mathrm{H}-{ }^{1} \mathrm{H}$ COSY and HMBC correlations of compounds $\mathbf{1}$ and 2

By comparing their physical and spectroscopic data with those reported in the literatures, the known compounds (Figure $\mathrm{S} 1)$ were elucidated as $(4 R, 6 R)$-carveol $\beta$-D-glucoside (3) [14], (4R,6S)-carveol $\beta$-D-glucoside (4) [14], (+)-neodihydrocarvy $\beta$-D-glucoside (5) [15], (-)-dihydrocarvy $\beta$-D-glucoside (6) [15], uroterpenol $\beta$-D-glucoside (7) [15], spicatoside A (8) [16], spicatoside B (9) [16], (3S,6S)-cislinalool-3,7-oxide (10) [17], $(3 R, 9 S)$-megastigman-5-en3,9-diol 3-O- $\beta$-D-glucopyranoside (11) [18], linarionoside A (12) [19], 1,1,5-trimethyl- 6-(3-hydroxyl) cyclohexene5-yl-1- $\beta$-D-pyranoglucoside (13) [20], linarionoside B (14) [21], (9S)-linarionoside B (15) [21], (+)-jasmololone glycoside (16) [14], (-)-5'-( $\beta$-D-glucopyranosyloxy) jasmonic acid (17) [22], maniladiol (18) [23], 3 $\beta, 28$ dihydroxy-olean-12-enyl palmitate (19) [24], olean-12-ene28-arboxy-3-palmitate (20) [25], ursolic acid (21) [26],
1-( $\beta$-D-ribofuranosyl)-1H-1,2,4-triazone (22) [27], naphthisoxazol A (23) [28], menthalactone (24) [29], 6-amino9-[1-(3,4-dihydroxy phenyl)ethyl]-9H-purine (25) [30], respectively.

\section{2 a-Glucosidase Inhibitory Activity}

In the preliminary bioassay, the crude extracts, Fr.A, Fr.C and Fr.D all exhibited significantly inhibitory activity against $\alpha$-glucosidase at concentrations $(>20 \mu \mathrm{g} / \mathrm{mL})$. These inhibitory effects were dose-dependent (Fig. 3). Their $\mathrm{IC}_{50}$ values were measured as 21.0, 36.7, 37.2, and $20.3 \mu \mathrm{g} / \mathrm{mL}$, respectively (Table 3). Bioactivity-guided isolation further afforded 25 compounds, while compounds 1-17 and 21-25 were measured their $\alpha$-glucosidase inhibitory activity. Compound 11 possessed the most significant activity with an $\mathrm{IC}_{50}$ values of $83.4 \mu \mathrm{M}$, while compounds $\mathbf{3}$ and $\mathbf{4}$ showed moderate inhibitory activity against $\alpha$-glucosidase with $\mathrm{IC}_{50}$ values of 516.0 and $919.0 \mu \mathrm{M}$, respectively (Table 3). Other compounds had no significant inhibitory activity.

Comparing the chemical structures and activity of these compounds, it can be found that triterpenoids ( 2 and $\mathbf{2 1}$ ), jasmonoid glucosides (16 and 17) and N-containing compounds (22-25) were not responsible for the $\alpha$-glucosidase inhibitory activity of the extracts from M. haplocalyx $(M$. canadensis). Among the monoterpene glucosides (1 and 3-10), only compounds $\mathbf{3}$ and $\mathbf{4}$ manifested moderate inhibitory activity against $\alpha$-glucosidase, while $\mathbf{5}$ and $\mathbf{6}$ did not. 
Table $2{ }^{1} \mathrm{H}$ NMR $\left(600 \mathrm{MHz}, \mathrm{CDCl}_{3}\right)$ and ${ }^{13} \mathrm{C}$ NMR $(150 \mathrm{MHz}$, $\mathrm{CDCl}_{3}$ ) data for compound $\mathbf{2}$

\begin{tabular}{|c|c|c|c|c|c|}
\hline No. & $\delta_{\mathrm{H}}$ & $\delta_{\mathrm{C}}$ & No. & $\delta_{\mathrm{H}}$ & $\delta_{\mathrm{C}}$ \\
\hline $1 \mathbf{a}$ & $1.96 \mathrm{~m}$ & $39.1 \mathrm{t}$ & 20 & & $31.1 \mathrm{~s}$ \\
\hline $1 b$ & $1.32 \mathrm{~m}$ & & $21 \mathrm{a}$ & $1.32 \mathrm{~m}$ & $34.9 \mathrm{t}$ \\
\hline $2 a$ & $1.62 \mathrm{~m}$ & $23.8 \mathrm{t}$ & $21 b$ & $1.10 \mathrm{~m}$ & \\
\hline $2 \mathbf{b}$ & $1.23 \mathrm{~m}$ & & $22 \mathrm{a}$ & $1.44 \mathrm{~m}$ & $37.0 \mathrm{t}$ \\
\hline 3 & $4.51 \mathrm{dd}(7.8,9.0)$ & $80.4 \mathrm{~d}$ & $22 b$ & $1.23 \mathrm{~m}$ & \\
\hline 4 & & $38.0 \mathrm{~s}$ & 23 & $0.85 \mathrm{~s}$ & $28.2 \mathrm{q}$ \\
\hline 5 & $0.88 \mathrm{~m}$ & $55.3 \mathrm{~d}$ & 24 & $0.90 \mathrm{~s}$ & $16.8 \mathrm{q}$ \\
\hline $6 \mathbf{a}$ & $1.52 \mathrm{~m}$ & $18.3 \mathrm{t}$ & 25 & $1.07 \mathrm{~s}$ & $16.8 \mathrm{q}$ \\
\hline $6 b$ & $1.26 \mathrm{~m}$ & & 26 & $1.00 \mathrm{~s}$ & $18.2 \mathrm{q}$ \\
\hline $7 \mathbf{a}$ & $1.50 \mathrm{~m}$ & $33.2 \mathrm{t}$ & 27 & $1.21 \mathrm{~s}$ & $25.1 \mathrm{q}$ \\
\hline $7 b$ & $1.30 \mathrm{~m}$ & & 28 & $0.83 \mathrm{~s}$ & $28.5 \mathrm{q}$ \\
\hline 8 & & $43.1 \mathrm{~s}$ & 29 & $0.89 \mathrm{~s}$ & $33.2 \mathrm{q}$ \\
\hline 9 & $1.73 \mathrm{~m}$ & $51.1 \mathrm{~d}$ & 30 & $0.88 \mathrm{~s}$ & $23.6 \mathrm{q}$ \\
\hline 10 & & $38.1 \mathrm{~s}$ & $\mathrm{OCH}_{3}$ & $3.25 \mathrm{~s}$ & $53.4 \mathrm{q}$ \\
\hline 11 & $3.89 \mathrm{dd}(3.0,9.0)$ & $75.8 \mathrm{~d}$ & $1^{\prime}$ & & $173.7 \mathrm{~s}$ \\
\hline 12 & $5.32 \mathrm{~d}(3.0)$ & $121.8 \mathrm{~d}$ & $2^{\prime}$ & $2.29 \mathrm{t}(7.8)$ & $34.7 \mathrm{t}$ \\
\hline 13 & & $149.7 \mathrm{~s}$ & $3^{\prime}$ & $1.64 \mathrm{~m}$ & $25.2 \mathrm{t}$ \\
\hline 14 & & $41.7 \mathrm{~s}$ & $4^{\prime}$ & & $29.2 \mathrm{t}$ \\
\hline $15 \mathbf{a}$ & $2.04 \mathrm{~m}$ & $26.3 \mathrm{t}$ & $5^{\prime}$ & & $29.3 \mathrm{t}$ \\
\hline $15 b$ & $0.84 \mathrm{~m}$ & & $6^{\prime}$ & & $29.4 \mathrm{t}$ \\
\hline $16 a$ & $1.65 \mathrm{~m}$ & $26.8 \mathrm{t}$ & $7^{\prime} \sim 13^{\prime}$ & & $29.6 \sim 29.7 \mathrm{t}$ \\
\hline $16 b$ & $1.00 \mathrm{~m}$ & & $14^{\prime}$ & $1.26 \mathrm{~m}$ & $31.9 \mathrm{t}$ \\
\hline 17 & & $32.3 \mathrm{~s}$ & $15^{\prime}$ & $1.27 \mathrm{~m}$ & $22.7 \mathrm{t}$ \\
\hline 18 & $2.01 \mathrm{dd}(13.8,4.2)$ & $47.0 \mathrm{~d}$ & $16^{\prime}$ & $0.88 \mathrm{t}(7.8)$ & $14.1 \mathrm{q}$ \\
\hline 19a & $1.66 \mathrm{dd}(13.8,4.2)$ & $46.5 \mathrm{t}$ & & & \\
\hline 19b & $1.08 \mathrm{~m}$ & & & & \\
\hline
\end{tabular}

Thus, it might be assumed that the absence of cyclic double bond in $\mathbf{3}$ and $\mathbf{4}$ markedly decreased their inhibitory activity.

\section{Experimental}

\subsection{General Experimental Instruments and Procedures}

LC-MS analyses were performed on a UFLC/MS-IT-TOF apparatus and the analytical conditions were set as previously reported [31]. Mass spectra were measured through a Waters AutoSpec Premier P776 (Waters, USA) mass spectrometer. Optical rotations were measured through a Jasco model 1020 digital polarimeter (Horiba, Tokyo, Japan). UV and IR (KBr) spectra were recorded on a Shimadzu UV2401PC spectrophotometer (Shimadzu, Kyoto, Japan) and a Bio-Rad FTS-135 spectrometer (Hercules, California, USA), respectively. NMR spectra were recorded on the DRX-500 or AdvanceIII-600 NMR (Bruker, Bremerhaven, Germany) spectrometers with TMS as an internal standard. Thin-layer chromatography (TLC) analyses were carried out with silica gel GF254 (Merck, Chemical Co. Ltd., Shanghai, China), and spots were detected under UV light or by heating after spraying with $10 \% \mathrm{H}_{2} \mathrm{SO}_{4}$ in $\mathrm{C}_{2} \mathrm{H}_{5} \mathrm{OH}(\mathrm{v} / \mathrm{v})$. Preparative TLC (PTLC) was purchased from Yantai Jiangyou silicon development Company (Yantai, China). Silica gel (200-300 mesh, Linyi Haixiang Co., Ltd; Linyi, China), Sephadex LH-20 (Amersham Bioscience, Sweden), and D101 macroporous adsorption resin (Tianjin guangfu fine

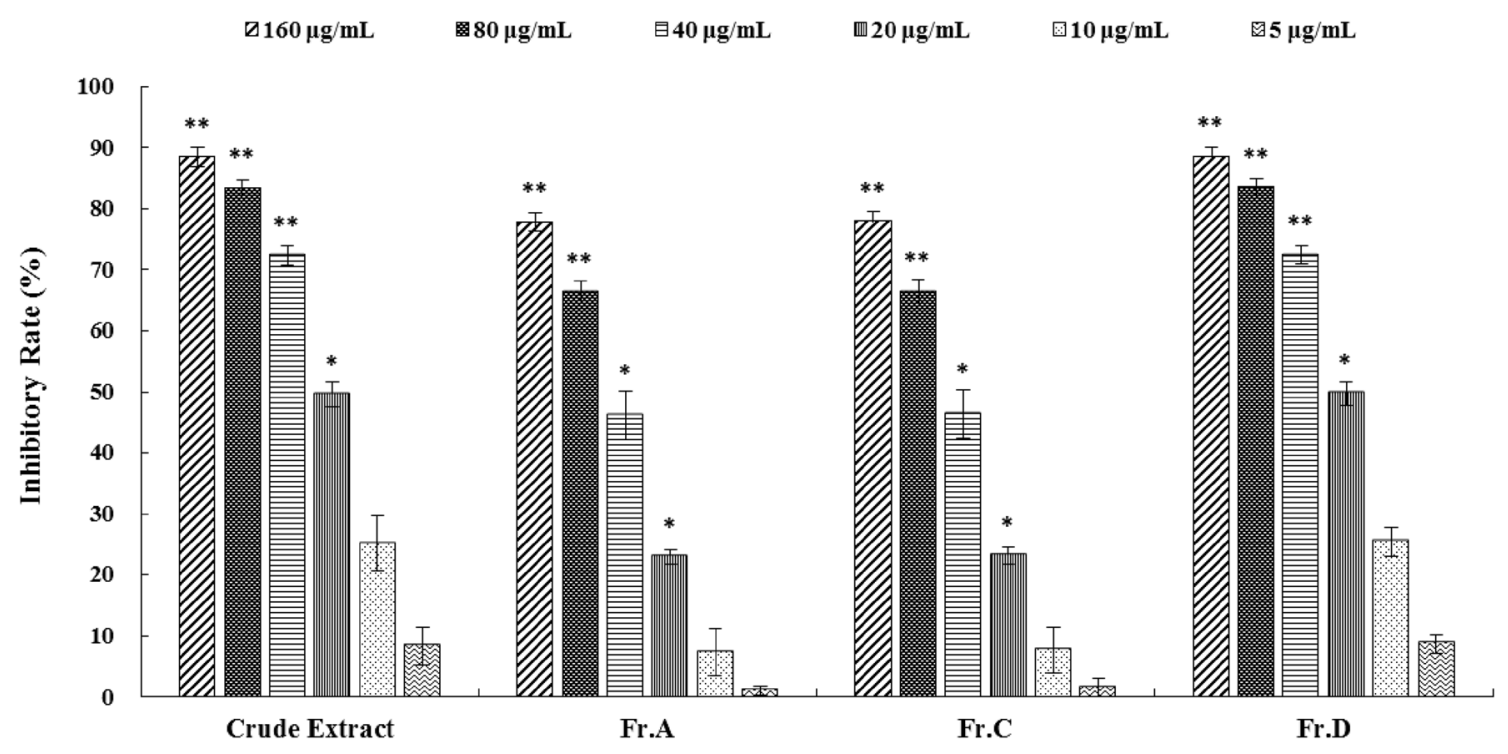

Fig. $3 \alpha$-Glucosidase inhibitory activities of crude extract, Fr.A, Fr.C and Fr.D at concentrations of $160,80,40,20,10$ and $5 \mu \mathrm{g} / \mathrm{mL}$, respectively. Values are presented as inhibitory rate compared to the blank control. Means significantly lower than the controls are indi- cated with one asterisk $(*)$ (Dunnett's one-sided t test; $\mathrm{p}<0.05$ ) or two asterisks $(* *)(\mathrm{p}<0.01)$. Error bars are one standard error of the mean. $\mathrm{N}=5$ 
Table $3 \alpha$-Glucosidase inhibitory activities of extracts and compounds from $M$. haplocalyx

\begin{tabular}{lccc}
\hline Extracts $^{\mathrm{a}}$ & $\mathrm{IC}_{50} \pm \mathrm{SD}$ & Compounds $^{\mathrm{b}}$ & $\mathrm{IC}_{50} \pm \mathrm{SD}$ \\
\hline Crude extract & $21.0 \pm 1.9$ & $\mathbf{3}$ & $516.0 \pm 2.1$ \\
Fr.A & $36.7 \pm 2.6$ & $\mathbf{4}$ & $919.0 \pm 37.3$ \\
Fr.C & $37.2 \pm 5.5$ & $\mathbf{1 1}$ & $83.4 \pm 1.3$ \\
Fr.D & $20.3 \pm 2.8$ & & \\
Acarbose $^{\mathrm{c}}$ & $25.8 \pm 3.3$ & & \\
\hline
\end{tabular}

${ }^{\mathrm{a}} \mathrm{IC}_{50}$ values in $\mu \mathrm{g} / \mathrm{mL}$ are mean $\pm \mathrm{SD}$ from three independent experiments

${ }^{\mathrm{b}} \mathrm{IC}_{50}$ values in $\mu \mathrm{M}$ are mean $\pm \mathrm{SD}$ from three independent experiments

${ }^{\mathrm{c}} \mathrm{IC}_{50}$ values in $\mathrm{nM}$ are mean $\pm \mathrm{SD}$ from three independent experiments

chemical co. LTD, Tianjin, China) were used for column chromatography. MPLC separations were conducted on a Dr-Flash II apparatus using a MCI gel CPH 20P (75-150 u, Mitsubishi Chemical Corporation, Tokyo, Japan) column. Semipreparative HPLC purifications were performed on a Shimadzu LC-CBM-20 system (Shimadzu, Kyoto, Japan) with the XDB-C18 column.

\subsection{Plant Material}

The aerial parts of fresh $M$. haplocalyx Briq. (M. canadensis L.) were bought from market, Kunming, Yunnan Province, China, in March 2013, and were identified by Prof. Li-gong Lei, Kunming Institute of Botany. A voucher specimen (No. 2013032401) was deposited at the Laboratory of Anti-virus and Natural Medicinal Chemistry, Kunming Institute of Botany, CAS.

\subsection{Extraction and Isolation}

The aerial parts of fresh M. haplocalyx (M. canadensis) $(20 \mathrm{~kg})$ were powdered and extracted with $50 \%$ ethanol for three times at room temperature (each $200 \mathrm{~L}$ ). The combined extraction was concentrated in vacuo to yield a residue. The residue was then suspended in water and extracted with ethyl acetate (Fr.A, $130 \mathrm{~g}$ ). The aqueous phase was subjected to D101 macroporous adsorption resin using a step gradient elution with $\mathrm{C}_{2} \mathrm{H}_{5} \mathrm{OH}-\mathrm{H}_{2} \mathrm{O}(0: 100,50: 50$ and $90: 10$, v/v) as the mobile phase to give three fractions (Fr.B-Fr.D).

The Fr.A (130 g) and Fr.D (10 g) were combined (Fr.E, $140 \mathrm{~g})$. Fr.E (140 g) was fractionated on silica gel CC using a step gradient elution with $\mathrm{MeOH}-\mathrm{CHCl}_{3}(2: 98$, $5: 95,10: 90, \mathrm{v} / \mathrm{v})$ and $\mathrm{H}_{2} \mathrm{O}-\mathrm{MeOH}-\mathrm{CHCl}_{3}$ (2:20:80, $3: 30: 70$. 4:40:60, v/v) as the mobile phase, and following washed with $\mathrm{MeOH}$ to give seven fractions (Fr.E-1-Fr.E7). Fr.E-3 (34 g) was subjected to silica gel CC eluted with EtOAc-petroleum ether (from 6:94 to 50:50) to give five fractions (Fr.E-3-1-Fr.E-3-5). Fr.E-3-1 (8.8 g) was submitted to silica gel $\mathrm{CC}$ eluted with $\mathrm{Me}_{2} \mathrm{CO}$-petroleum ether (5:95), then applied to Sephadex LH-20 CC eluted with $\mathrm{CHCl}_{3}-\mathrm{MeOH}$ (50:50), and finally purified by silica gel CC with EtOAc-petroleum ether (40:60) to yield 2 (10 mg), $19(13 \mathrm{mg})$ and $20(12 \mathrm{mg})$. Compound 21 (2 g) was got from Fr.E-3-2 (9.1 g) that was separately separated on MCI gel CHP 20P CC $\left(\mathrm{MeOH}-\mathrm{H}_{2} \mathrm{O}, 30: 70\right.$, 70:30, 90:10) and Sephadex LH-20 CC (MeOH-CHCl 3 , 50:50). Fr.E-3-5 (5.6 g) was subjected on MCI gel CHP 20P CC using $\mathrm{MeOH}-\mathrm{H}_{2} \mathrm{O}$ (60:40, 90:10), subsequently compound 24 (10 mg) was yielded from Sephadex LH-20 CC (MeOH-CHCl 3 , 50:50). Fr.E-5 (5.8 g) was subjected to MCI gel CHP 20P CC using a step gradient elution with $\mathrm{MeOH}-\mathrm{H}_{2} \mathrm{O}(40: 60,80: 20)$ to give two fractions (Fr.A-5-1 and Fr.A-5-2). Fr.A-5-1 (2.7 g) was fractionated on silica gel $\mathrm{CC}\left(\mathrm{MeOH}-\mathrm{CHCl}_{3}, 10: 90\right)$ to give three fractions (from Fr.E-5-1-1 to Fr.E-5-1-3). Fr.E-5-1-1 (327 mg) was preferred on Sephadex LH-20 eluted with $\mathrm{MeOH}-\mathrm{CHCl}_{3}(50: 50)$, and following purified by semipreparative $\mathrm{HPLC}\left(\mathrm{MeCN}-\mathrm{H}_{2} \mathrm{O}, 25: 75\right.$, v/v, $\left.3.0 \mathrm{~mL} / \mathrm{min}\right)$ over an XDB-C ${ }_{18}$ column $(9.4 \times 250 \mathrm{~mm}, 5 \mu \mathrm{m})$ to yield compounds 3 (5 mg), 5 (4 mg), 6 (5 mg) and 25 (8 mg). Fr.E-5-1-2 (1.3 g) was separated on Sephadex LH-20 $\mathrm{CC}\left(\mathrm{MeOH}-\mathrm{CHCl}_{3}, 50: 50\right)$, and later fractionated on silica gel $\mathrm{CC}\left(\mathrm{MeOH}-\mathrm{CHCl}_{3}, 90: 10\right)$, therefore, compounds 1 (4 mg), 7 (14 mg), 10 (2 mg), the mixture of 12 and $\mathbf{1 3}$ ( $8 \mathrm{mg})$, the mixture of $\mathbf{1 4}$ and $\mathbf{1 5}(6 \mathrm{mg})$ were obtained by semipreparative HPLC $\left(\mathrm{MeCN}_{-} \mathrm{H}_{2} \mathrm{O}, 40: 60\right.$, v/v, $3.0 \mathrm{~mL} /$ $\mathrm{min})$ over an $\mathrm{XDB}-\mathrm{C}_{18}$ column $(9.4 \times 250 \mathrm{~mm}, 5 \mu \mathrm{m})$.

Fr.C (50 g) was subjected to silica gel $\mathrm{CC}$ and then $\mathrm{Al}_{2} \mathrm{O}_{3} \mathrm{CC}$ with $\mathrm{H}_{2} \mathrm{O}-\mathrm{MeOH}-\mathrm{CHCl}_{3}(3: 30: 70)$ to give four fractions (from Fr.C-1 to Fr.C-4). Fr.C-1 (10.4 g) was fractionated on MCI gel CHP 20P CC with $\mathrm{MeOH}-\mathrm{H}_{2} \mathrm{O}$ (40:60, 80:20), then applied to Sephadex LH-20 CC eluted with $\mathrm{MeOH}-\mathrm{CHCl}_{3}$ (50:50), and later purified by silica gel $\mathrm{CC}$ eluted with $\mathrm{MeOH}-\mathrm{EtOAc}(2: 98)$ to yield 4 (120 mg), 8 (800 mg), 9 (311 mg), $11(200 \mathrm{mg})$ and 16 (20 mg). Compounds 17 (201 mg), 22 (46 mg) and $\mathbf{2 3}$ (17 mg) were afforded from Fr.C-2 (20.0 g) by repeated silica gel CC $\left(\mathrm{MeOH}-\mathrm{CHCl}_{3}\right.$, 15:85, 20:80, 25:75) and Sephadex LH-20 $\left(\mathrm{MeOH}-\mathrm{CHCl}_{3}, 50: 50\right)$.

\subsection{Spectroscopy Data of Compounds}

\subsubsection{Compound 1}

Colorless caramelized solid, $\mathrm{C}_{16} \mathrm{H}_{24} \mathrm{O}_{7},[\alpha]_{\mathrm{D}}^{20}-22.0(c$, $0.20, \mathrm{MeOH})$; UV (MeOH) $\lambda_{\text {max }}(\log \varepsilon): 230$ (3.65) nm; IR (KBr) $\nu_{\max }: 3427,1635,1385,1046,902 \mathrm{~cm}^{-1} ;{ }^{1} \mathrm{H} \mathrm{NMR}$ (600 MHz, CD 3 OD) and ${ }^{13} \mathrm{C}$ NMR (DEPT, $150 \mathrm{MHz}$, 
$\left.\mathrm{CD}_{3} \mathrm{OD}\right)$ see Table 1; HRESIMS $m / z: 351.1404[\mathrm{M}+\mathrm{Na}]^{+}$ (calc. 351.1414 for $\mathrm{C}_{16} \mathrm{H}_{24} \mathrm{O}_{7} \mathrm{Na}$ ).

\subsubsection{Compound 2}

Colorless powder, $\mathrm{C}_{47} \mathrm{H}_{82} \mathrm{O}_{3} ;{ }^{1} \mathrm{H}$ NMR (600 MHz, $\mathrm{CDCl}_{3}$ ) and ${ }^{13} \mathrm{C}$ NMR (DEPT, $150 \mathrm{MHz}, \mathrm{CDCl}_{3}$ ) see Table 2; HREIMS $m / z$ : $694.6258[\mathrm{M}]^{+}$(calc. 694.6264 for $\mathrm{C}_{47} \mathrm{H}_{82} \mathrm{O}_{3}$ ).

\subsection{Inhibitory Assay of a-Glucosidase}

The $\alpha$-glucosidase inhibitory activity was measured in a 96-well microtiter plate based on p-nitrophenyl- $\alpha$-Dglucopyranoside (PNPG, Yuanye Biosciences Co. Ltd., Shanghai, China) as a substrate following the reported method with slight modifications [32]. In brief, $5.0 \mathrm{mM}$ PNPG $(20 \mu \mathrm{L})$ and $20 \mu \mathrm{L}$ tested compounds of dissolved in $10 \mu \mathrm{L}$ DMSO and $990 \mu \mathrm{L}$ phosphate buffer $(\mathrm{PB}, 0.1 \mathrm{M}$, $\mathrm{pH}=6.8$ ) were sequentially added to a 96-well plate to be mixed. The mixture was incubated at $37^{\circ} \mathrm{C}$ for $5 \mathrm{~min}$. Reactions were initiated by addition of $2.0 \mathrm{U} / \mathrm{mL} \alpha$-glucosidase (20 $\mu \mathrm{L}$, Yuanye Biosciences Co. Ltd., Shanghai, China) in PB. The reaction mixture was incubated at $37^{\circ} \mathrm{C}$ for $15 \mathrm{~min}$. Then, the incubation solution was stopped the reaction by adding $0.2 \mathrm{M} \mathrm{Na}_{2} \mathrm{CO}_{3}(40 \mu \mathrm{L})$. The absorbance was recorded at $405 \mathrm{~nm}$ by a Bio-Rad 680 microplate reader (Hercules, CA, USA). The negative control was set by adding $\mathrm{PB}$ instead of the sample using the same procedure for the tests. Acarbose (Bayer) dissolved in PB was utilized as the positive control. The blank was set by adding phosphate buffer instead of the $\alpha$-glucosidase using the same method. Inhibition rate $(\%)=[($ ODnegative control - ODblank) - (ODtest - ODtest blank) $] /$ (ODnegative blank - ODblank) $\times 100 \%$. All data were subjected to an analysis of variance using SPSS 18.0. The significant differences in inhibition rates between the treatment and blank control were calculated using one-way analysis of variance (ANOVA).

\section{Conclusion}

In this study, the extracts of the aerial parts of $M$. haplocalyx (M. canadensis) were firstly found to exhibit significantly inhibitory activity against $\alpha$-glucosidase. Two new compounds ( $\mathbf{1}$ and $\mathbf{2}$ ) and 23 known ones were isolated and identified through bioactivity-guided fractionation. Among them, compounds 3-9 and $\mathbf{2 4}$ were reported from M. haplocalyx (M. canadensis) for the first time, while compounds 10-20, 22-23 and 25 were firstly isolated from the genus Mentha. Bioactivity assay further traced the active compounds $(3,4$ and 11), whose inhibitory activity against $\alpha$-glucosidase had not been reported before. It was noted that the monoterpene glucosides and the ionone glycosides endowed this plant with the $\alpha$-glucosidase inhibitory activity.

Acknowledgements This work was supported by the CAS Hundred Talents Program, the Youth Innovation Promotion Association of the Chinese Academy of Sciences (CN), and Southeast Asia Biodiversity Research Institute, CAS (2017CASSEABRIQG003).

\section{Compliance with Ethical Standards}

Conflict of interests No potential conflict of interest was reported by the authors.

Open Access This article is distributed under the terms of the Creative Commons Attribution 4.0 International License (http://creativeco mmons.org/licenses/by/4.0/), which permits unrestricted use, distribution, and reproduction in any medium, provided you give appropriate credit to the original author(s) and the source, provide a link to the Creative Commons license, and indicate if changes were made.

\section{References}

1. M.R. Bhandari, N. Jong-Anurakkun, G. Hong, J. Kawabata, Food Chem. 106, 247-252 (2008)

2. H. Wang, Y.J. Du, H.C. Song, Food Chem. 123, 6-13 (2010)

3. K.Y. Kim, K.A. Nam, H. Kurihara, S.M. Kim, Phytochemistry 69, 2820-2825 (2008)

4. K.Y. Kim, M.H. Wang, H.I. Rhee, Carbohydr. Res. 339, 715-717 (2004)

5. Y.Q. Li, F.C. Zhou, F. Gao, J.S. Bian, F. Shan, J. Agric. Food Chem. 57, 11463-11468 (2009)

6. B.T. Zhao, T.I. Kim, Y.H. Kim, J.S. Kang, B.S. Min, J.K. Son, M.H. Woo, Nat. Prod. Res. 32, 239-242 (2018)

7. G. Cao, Q. Shan, X. Li, X. Cong, Y. Zhang, H. Cai, B. Cai, Analyst 136, 4653-4661 (2011)

8. G.M. She, C. Xu, B. Liu, R.B. Shi, Helv. Chim. Acta 93, 2495$2498(2010)$

9. S.A. Abdellatief, R.R. Beheiry, E.M. Sam, Biomed. Pharmacother. 95, 990-999 (2017)

10. S.M. Barbalho, F.M.V.F. Machado, M. Oshiiwa, M. Abreu, E.L. Guiger, P. Tomazela, R.A. Goulart, Food Sci. Technol. 31, 584588 (2011)

11. R. Lakshmi, T.D. Bateman, M.C. McIntosh, J. Org. Chem. 70, 5313-5315 (2005)

12. S.H. Park, K.S. Kim, Y. Suzuki, S.U. Kim, Phytochemistry 44, 623-626 (1997)

13. C.Y. Ragasa, D.L. Espineli, C.C. Shen, Nat. Prod. Res. 26, 1869$1875(2012)$

14. S. Yamamura, K. Ozawa, K. Ohtani, R. Kasai, K. Yamasaki, Phytochemistry 48, 131-136 (1998)

15. S. Shimizu, J. Essent. Oil Res. 2, 81-86 (1990)

16. J. Zheng, L.J. Wu, L. Zheng, B. Wu, A.H. Song, J. Asian Nat. Prod. Res. 5, 69-73 (2003)

17. L.H. Jiang, H. Kojima, K. Yamada, A. Kobayashi, K. Kubota, J. Agri. Food Chem. 49, 5888-5894 (2001)

18. T. Nakanishi, N. Iida, Y. Inatomi, H. Murata, A. Inada, J. Murata, F.A. Lang, M. Iinuma, Y. Sakagami, Chem. Pharm. Bull. 53, 783-787 (2005)

19. L.H. Yan, L.Z. Xu, Z.M. Wang, Q.W. Zhang, S.L. Yang, Acta Pharm. Sin. 45, 1527-1532 (2010)

20. G.Q. Li, Z.J. Jia, S.S. Zhang, Chem. Res. Chinese Univ. 19, 422424 (2003) 
21. S. Lin, Y.L. Zhang, M.T. Liu, J.C. Zi, M.L. Gan, W.X. Song, X.N. Fan, S.J. Wang, Y.C. Yang, J.G. Shi, China J. Chin. Mater Med. 40, 2602-2611 (2015)

22. T. Fujita, K. Terato, M. Nakayama, Biosci. Biotechnol. Biochem. 60, 732-735 (1996)

23. S. Lin, Z.X. Zhang, H.H. Shen, H.L. Li, L. Shan, R.H. Liu, X.K. Xu, W.D. Zhang, China J Chin Mater Med. 35, 1137-1141 (2010)

24. M.L. Barreiros, J.M. David, P.A.P. de Pereira, M.L.S. Guedes, J.P. David, J. Brazil. Chem. Soc. 13, 669-673 (2002)

25. L. Barbosa, F.L. Mathias, R. Braz-Filho, I.J.C. Vieira, J. Brazil. Chem. Soc. 21, 1434-1438 (2010)

26. M.G.V. Silva, Í.G.P. Vieira, F.N.P. Mendes, I.L. Albuquerque, R.N. dos Santos, F.O. Silva, S.M. Morais, Molecules 13, 2483 2487 (2008)
27. R.M. Huang, X.F. Zhou, Y. Peng, X.W. Yang, T.H. Xu, Y.H. Liu, Chem. Nat. Compd. 46, 1010-1011 (2011)

28. G.Q. Li, Y.B. Zhang, H.S. Guan, Fitoterapia 79, 238-239 (2008)

29. L.I.M. Villaseñor, A.C. Sanchez, Z Naturforsch C: J. Biosciences. 64, 809-812 (2009)

30. H.Y. Ma, Y. Sun, Y.Z. Zhou, M. Hong, Y.H. Pei, Molecules 13, 267-271 (2008)

31. C.A. Geng, H. Chen, X.L. Chen, X.M. Zhang, L.G. Lei, J.J. Chen, I. Int, J. Mass Spectrom. 361, 9-22 (2014)

32. Y. Luo, Q.L. Xu, L.M. Dong, Z.Y. Zhou, Y.C. Chen, W.M. Zhang, J.W. Tan, Phytochem. Lett. 11, 127-131 (2015) 